
ПРИ САХАРНОМ ДИАБЕТЕ

() Н.Н. Мусина ${ }^{1 *}$, Т.В. Саприна', Т.С. Прохоренко ${ }^{1,2}$, А.П. Зима'

${ }^{1}$ Сибирский государственный медицинский университет, Томск, Россия

${ }^{2}$ Томский региональный центр крови, Томск, Россия

ОБОСНОВАНИЕ. Известно, что метаболические нарушения при сахарном диабете имеют регулирующее влияние на феррокинетику, в связи с чем сахарный диабет часто сопровождается различными нарушениями обмена железа как анемией, так и вторичной перегрузкой железом. Основной проблемой является своевременная и точная дифференциальная диагностика между анемией хронических заболеваний и железодефицитной анемией. Для решения данной проблемы необходимо установление надежных лабораторных маркеров анемии хронических заболеваний, а также понимание того, какие метаболические нарушения могут приводить к возникновению и утяжелять течение данного вида анемии.

ЦЕЛЬ. Изучить частоту встречаемости нарушений феррокинетики у пациентов с сахарным диабетом, а также установить клинико-биохимические корреляции, значимые в дифференциальной диагностике различных нарушений обмена железа - железодефицитной анемии, анемии хронических заболеваний и синдрома дисметаболической перегрузки железом - при сахарном диабете.

МЕтоДЫ. Дизайн исследования - одномоментное обсервационное одноцентровое. Исследование проводилось на базе эндокринологической клиники ФГБОУ ВО СибГМУ г. Томска. В исследование были включены 76 пациентов с сахарным диабетом 1 и 2 типа. Всем пациентам проводились: оценка антропометрических данных; исследование гликированного гемоглобина; исследование уровня креатинина с расчетом скорости клубочковой фильтрации (СКФ) по формуле CKD-EPI. Также оценивались количество эритроцитов, ретикулоцитов, концентрация гемоглобина, уровень гематокрита и биохимические показатели обмена железа - концентрации сывороточного железа и ферритина; проводилась оценка концентрации гепсидина и неспецифических маркеров воспаления - скорости оседания эритроцитов (СОЭ) и высокочувствительного С-реактивного белка (СРБ).

РЕЗУЛЬТАТЫ. Из 76 включенных в исследование пациентов 20 человек (26,3\%) страдали сахарным диабетом 1 типа и 56 (73,3\%) - сахарным диабетом 2 типа. Параметры феррокинетики значимо не различались у пациентов с сахарным диабетом 1 и 2 типа, при этом в группе пациентов с сахарным диабетом 2 типа уровни СРБ (р=0,034) и лейкоцитов крови $(p=0,020)$ были достоверно выше, чем у пациентов с сахарным диабетом 1 типа. Как в общей выборке пациентов с нарушениями углеводного обмена, так и у пациентов с сахарным диабетом 2 типа в структуре анемического синдрома преобладала анемия хронических заболеваний. После разделения общей выборки пациентов на группы по виду анемического синдрома - отсутствие анемии, анемия хронических заболеваний и железодефицитная анемия - был проведен сравнительный анализ средних значений маркеров воспаления и уровня гепсидина в данных группах. Было установлено, что у пациентов с анемией хронических заболеваний уровень гепсидина достоверно выше, чем у пациентов без анемического синдрома $(p=0,033)$. Парный корреляционный анализ показал наличие положительной корреляции СОЭ с микроальбуминурией $(r=0,515 ; \mathrm{p}<0,0001)$, уровнем креатинина $(r=0,467 ; p<0,0001)$ и отрицательной - c СКФ $(r=-0,436 ; p<0,0001)$ и железом сыворотки $(r=-0,276 ; p=0,017)$. В результате ROC-анализа наибольшую информативность в диагностике анемии хронических заболеваний показали: ферритин - чувствительность 78\%, специфичность 52\% при диагностическом пороге 75,5 нг/мл (площадь под кривой 0,695; p=0,006); СОЭ - чувствительность 67\%, специфичность 64\% при диагностическом пороге 15,5 мм/ч (площадь под кривой 0,750; $p=0,040$ ) и СРБ - чувствительность 67\%, специфичность 64\% при диагностическом пороге 5,2 нг/мл (площадь под кривой 0,646; $p<0,0001$ ).

ЗАКЛЮЧЕНИЕ. Таким образом, исследованные маркеры воспаления - СОЭ и СРБ, а также гепсидин в совокупности с классическим диагностическим параметром - ферритином продемонстрировали высокую ценность в диагностике анемии хронических заболеваний и могут быть внесены в модифицированный алгоритм дифференциальной диагностики анемического синдрома у пациентов с сахарным диабетом.

КЛЮЧЕВЫЕ СЛОВА: сахарный диабет, анемия хронических заболеваний, железодефицитная анемия, гепсидин, С-реактивный белок, воспаление. 


\title{
SEARCHING FOR ADDITIONAL MARKERS OF IMPAIRED IRON METABOLISM IN DIABETES MELLITUS
}

\author{
(c) Nadezhda N. Musina1* ${ }^{1 *}$ Tatiana V. Saprina ${ }^{1}$, Tatiana S. Prokhorenko ${ }^{1,2}$, Anastasia P. Zima ${ }^{1}$
}

'Siberian State Medical University, Tomsk, Russia

${ }^{2}$ Tomsk Regional Blood Center, Tomsk, Russia

BACKGRAUND: It is known that metabolic disorders in diabetes mellitus have a regulating effect on ferrokinetics, and therefore diabetes mellitus is often accompanied by various disorders of iron metabolism, both anemia and secondary iron overload. The main problem is timely and accurate differential diagnosis between anemia of chronic diseases and iron deficiency anemia. It is necessary to establish reliable laboratory markers of anemia of chronic diseases in order to solve this problem, as well as to understand what metabolic disorders can lead to the occurrence and aggravate the course of this type of anemia.

AIMS: To study the frequency of occurrence of violations of ferrokinetics in patients with diabetes mellitus, as well as to establish clinical and biochemical correlations that are significant in the differential diagnosis of various disorders of iron metabolism: iron deficiency anemia, anemia of chronic diseases and dysmetabolic iron overload syndrome in diabetes mellitus.

MATERIALS AND METHODS: The research design a single-stage observational single-center research. The research was conducted on the basis of the endocrinological clinic of the Federal State Budgetary Educational Institution of Higher Education Siberian State Medical University in Tomsk. The research included 76 patients with type 1 and type 2 diabetes mellitus. We conducted an assessment of all patients as follows: anthropometric data assessment; glycated hemoglobin study; creatinine level study with the calculation of glomerular filtration rate (GFR) using the CKD-EPI formula. We also evaluated the number of erythrocytes, reticulocytes, the hemoglobin concentration, haematocrit level and biochemical parameters of iron metabolism: serum iron and ferritin concentrations; the concentration of hepsidin and non-specific markers of inflammation: erythrocyte sedimentation rate (ESR) and highly sensitive C-reactive protein (CRP).

RESULTS: 20 people (26.3\%) of the 76 patients included in the study, had type 1 diabetes mellitus and 56 people (73.3\%) had type 2 diabetes mellitus. The parameters of ferrokinetics did not significantly differ in patients with type 1 and type 2 diabetes mellitus, while in the group of patients with 20 patients (26.3\%) from the 76 ones included into the research had type 1 diabetes mellitus and 56 (73.3\%) from them had type 2 diabetes mellitus. The parameters of ferrokinetics did not significantly differ in patients with type 1 and type 2 diabetes mellitus, while in the group of patients with type 2 diabetes mellitus, the levels of CRP $(p=0.034)$ and blood leukocytes $(p=0.020)$ were significantly higher than in patients with type 1 diabetes mellitus. Both in the main group of patients with impaired carbohydrate metabolism, and in patients with type 2 diabetes mellitus, anemia of chronic diseases prevailed in the structure of the anemia syndrome. After dividing the main group of patients into groups by type of anemia syndrome: absence of anemia, anemia of chronic diseases and iron deficiency anemia, a comparative analysis of the average values of markers of inflammation and the level of hepsidin in these groups was performed. It was found that in patients with anemia of chronic diseases, the level of hepsidin is significantly higher than in patients without anemic syndrome $(p=0.033)$. Paired correlation analysis showed a positive correlation of ESR with microalbuminuria $(r=0.515 ; P<0.0001)$, creatinine level $(r=0.467 ; P<0.0001)$ and negative - with $G F R(r=-0.436 ; P<0.0001)$ and iron in serum $(r=-0.276 ; p=0.017)$. As the result of ROC analysis the most informative in the diagnosis of anemia of chronic disease were: ferritin - sensitivity $78 \%$, specificity $52 \%$ with a diagnostic threshold of $75.5 \mathrm{ng} / \mathrm{ml}$ (area under the curve 0,$695 ; \mathrm{p}=0.006$ ); ESR - sensitivity $67 \%$, specificity $64 \%$ with a diagnostic threshold of $15.5 \mathrm{~mm} / \mathrm{HR}$ (area under the curve of $0.750 \mathrm{in} ; \mathrm{p}=0.040$ ) and the CRP — sensitivity $67 \%$, specificity $64 \%$ with a diagnostic threshold of $5.2 \mathrm{ng} / \mathrm{ml}$ (area under the curve 0,$646 ; p<0.0001$ ).

CONCLUSION: Thus, the studied markers of inflammation - ESR and CRP, as well as hepsidin in combination with the classic diagnostic parameter - ferritin, demonstrated high value in the diagnosis of anemia of chronic diseases and can be included in the modified algorithm for differential diagnosis of anemia syndrome in patients with diabetes mellitus.

KEYWORDS: diabetes mellitus, anemia of chronic diseases, iron deficiency anemia, hepsidin, C-reactive protein, inflammation.

\section{ОБОСНОВАНИЕ}

Известно, что метаболические нарушения при сахарном диабете имеют, в том числе, и регулирующее влияние на феррокинетику [1], в связи с чем сахарный диабет часто сопровождается различными нарушениями обмена железа - как анемией, так и вторичной перегрузкой железом $[2,3,4]$. Анемия является распространенным и часто несвоевременно диагностируемым осложнением сахарного диабета 1 и 2 типа. При высокой частоте развития анемии у пациентов с нарушениями углеводного обмена этиология и патогенез ее различны. При этом основной проблемой является своевременная и точная дифференциальная диагностика между анемией хронических заболеваний (называемой еще анемией воспаления или железоперераспределительнойанемией) ижелезодефицитной анемией. Для решения данной проблемы необходимо установление надежных лабораторных маркеров анемии хронических заболеваний, а также понимание того, какие метаболические нарушения могут приводить к возникновению и утяжелять течение данной анемии. В последние годы особое внимание уделяется роли гепсидина в регуляции метаболизма железа. Согласно данным литературы, у пациентов с сахарным диабетом продукция гепсидина 
может снижаться [1], сопровождаясь синдромом перегрузки железом, или повышаться и приводить к развитию анемии хронических заболеваний [2-4]. Известно, что сахарный диабет и ожирение относятся к состояниям, сопровождающимся хроническим системным воспалением и повышением уровней провоспалительных маркеров интерлейкинов (IL-1 $\beta$, IL-6), C-реактивного белка (СРБ), активирующих сигнальный путь JAK/STAT с повышением экспрессии гена НАMР и синтеза гепсидина, что приводит к развитию анемии хронических заболеваний [5, 6]. Кроме указанного механизма развития анемии при сахарном диабете, возможной причиной нарушения синтеза гепсидина является хроническая болезнь почек. С потерей функционирующей паренхимы почек и снижением клиренса креатинина регистрируется одновременное снижение продукции эритропоэтина, который в норме подавляет образование гепсидина гепатоцитами, что способствует повышению уровня данного пептида в крови [7]. Таким образом, изучение структуры дизрегуляции обмена железа при диабете, уточнение особенностей патогенеза анемии хронических заболеваний, развившейся и протекающей на фоне сахарного диабета, а также совершенствование методов диагностики нарушений феррокинетики у лиц с патологией углеводного обмена представляется актуальной задачей.

\section{ЦЕЛЬ}

Изучить частоту встречаемости нарушений феррокинетики у пациентов с сахарным диабетом, а также установить клинико-биохимические корреляции, значимые в дифференциальной диагностике различных нарушений обмена железа - сидеропенической анемии, анемии хронических заболеваний и синдрома дисметаболической перегрузки железом - при сахарном диабете.

\section{МЕТОДЫ}

Дизайн исследования

Дизайн исследования - одномоментное обсервационное одноцентровое исследование.

\section{Критерии соответствия}

Критериями включения в исследование являлись: наличие установленного диагноза сахарного диабета 1 или 2 типа, возраст пациента от 18 до 70 лет, стаж заболевания от 1 года до 30 лет, уровень гликированного гемоглобина 6,5\% и выше, скорость клубочковой фильтрации (СКФ) по CKD-EPI выше 15 мл/мин (стадия хронической болезни почек (ХБП) С1-С4). Критерии исключения: инфекционные заболевания в стадии обострения - такие заболевания, как ВИЧ/СПИД, вирусные гепатиты с любой степенью активности, цирроз печени вирусной и аутоиммунной этиологии, туберкулез; онкологические заболевания; наличие в анамнезе гемотрансфузий в период 1 месяца до включения в исследование и во время исследования; пред- и постоперационный период; острая почечная, печеночная, сердечная недостаточность, СКФ ниже 15 мл/мин, стадия протеинурии; декомпенсация сахарного диабета с наличием кетоацидоза/осмотической дегидратации; прием препаратов железа; отказ пациента от участия в исследовании, неподписанное информированное согласие.

\section{Условия проведения}

Исследование проводилось на базе эндокринологической клиники ФГБОУ ВО СибГМУ г. Томска. В исследование были включены 76 пациентов с сахарным диабетом.

Продолжительность исследования

Набор пациентов в исследование проводился в течение одного года (2018-2019 гг.). В исследование включались пациенты, находящиеся на плановой госпитализации в эндокринологической клинике.

Описание медицинского вмешательства

Материалом исследования являлись сыворотка и плазма венозной крови, взятые утром натощак из локтевой вены в количестве 10 мл в вакуумные пробирки BD Vacutainer.

Исходы исследования

В результате исследования планируется установить частоту и структуру нарушений обмена железа у пациентов с сахарным диабетом 1 и 2 типов (железодефицитная анемия, анемия хронических заболеваний, дисметаболическая перегрузка железом). Планируется исследовать и сравнить основные гематологические и биохимические показатели обмена железа, такие как количество эритроцитов, ретикулоцитов, концентрация гемоглобина, уровень гематокрита, концентрация сывороточного железа, ферритина и гепсидина у пациентов с сахарным диабетом 1 и 2 типа. Также планируется исследовать и сравнить концентрации маркеров воспаления, таких как скорость оседания эритроцитов и высокочувствительный С-реактивный белок у пациентов с сахарным диабетом 1 и 2 типа. Планируется установить информативность гепсидина, СРБ и скорости оседания эритроцитов (СОЭ) в дифференциальной диагностике анемического синдрома у пациентов с сахарным диабетом.

\section{Методы регистрации исходов}

Всем пациентам проводились: оценка антропометрических данных; исследование гликированного гемоглобина как основного параметра оценки компенсации углеводного обмена - с использованием анализатора D10 (BIO-RAD, США); исследование уровня креатинина с использованием анализатора ARCHITECT i2000SR (Abbott, CША) с расчетом CKФ по формуле CKD-EPI. Также оценивались основные гематологические показатели (количество эритроцитов, ретикулоцитов, концентрация гемоглобина, уровень гематокрита) - с использованием гематологического анализатора XN1000 (Sysmex, Япония), а также биохимические показатели обмена железа - концентрация сывороточного железа (мкмоль/л) и ферритина (нг/мл) - с использованием анализатора ARCHITECT i2000SR (Abbott, США). Также среди всех включенных в исследование пациентов проводилась оценка концентрации гепсидина (пг/мл) методом иммуноферментного анализа (ИФА) (Cloud Clone, США), а также маркеров воспаления - СОЭ гематологическим анализатором XN1000 (Sysmex, Япония) и высокочувствительного СРБ (нг/мл) методом иммуноферментного анализа (ИФА) («Вектор Бест», Россия). Диагноз анемии устанавливался в соответствии с Федеральными клиническими рекомендациями по диагностике и лечению железодефицитной анемии (Румянцев А.Г., Масчан А.А. и др., 2015 г.) [8] 
и Федеральными клиническими рекомендациями по диагностике и лечению анемии хронических болезней (Румянцев А.Г., Масчан А.А., 2014 г.) [9].

\section{Анализ в подгруппах}

Все включенные в исследование пациенты были разделены на две группы: первая - пациенты с сахарным диабетом 1 типа и вторая - пациенты с сахарным диабетом 2 типа. Данное исследование не предполагало группы контроля.

\section{Этическая экспертиза}

Все включенные в исследование пациенты подписали добровольное информированное согласие. Протокол исследования был одобрен локальным этическим комитетом ФГБОУ ВО СибГМУ Минздрава России, номер протокола 5596 от 06.11.2017 г.

\section{Статистический анализ}

Статистическая обработка результатов осуществлялась с использованием пакета программ SPSS Statistics 23. С целью установления объема выборки был использован калькулятор расчета выборки, при выборе доверительного интервала, равного 5, необходимый размер репрезентативной выборки составил от 70 человек. Также использовалась последовательная стратегия расчета объема выборки с учетом коэффициента вариации (=среднеквадратичное отклонение от среднеарифметического в процентах), по результатам которой необходимый объем выборки составил от 61 человека. Проверка нормальности распределения проводилась по критерию Колмогорова-Смирнова. Пороговый уровень значимости (р) был принят равным 0,05. Полученные данные возраста, стажа заболевания, а также гликированного гемоглобина, индекса массы тела и микроальбуминурии не подчинялись нормальному закону распределения и были представлены в виде медианы и интерквартильного размаха (Me, $\left.\mathrm{Q}_{0,25}-\mathrm{Q}_{0,75}\right)$. Показатели феррокинетики и маркеров воспаления подчинялись нормальному закону распределения и были представлены в виде среднего и стандартного отклонения $(\mathrm{m} \pm \sigma)$, проверка нормальности распределения проводилась по критерию Колмогорова-Смирнова. В целях установления значимости различий показателей между независимыми группами сравнения была проведена статистическая обработка с помощью критерия Манна-Уитни для двух групп. Для показателей, имевших нормальное распределение (показатели обмена железа, СОЭ и СРБ), проводилась статистическая обработка с помощью критерия Стьюдента. Корреляцию между признаками оценивали с помощью критерия Спирмена для непараметрических данных. Пороговый уровень значимости был принят равным 0,05. Статистическую значимость различий частоты встречаемости качественных признаков в анализируемых группах проверяли с помощью критерия $\mathrm{X}^{2}$ Пирсона с критическим уровнем значимости $\mathrm{p}<0,05$.

\section{РЕЗУЛЬТАТЬ}

Объекты (участники) исследования

Из 76 включенных в исследование пациентов 20 человек (26,3\%) страдали сахарным диабетом 1 типа и 56 человек (73,3\%) - сахарным диабетом 2 типа. Среди включенных в исследование пациентов было 28 (36,8\%) мужчин и 48 (63,2\%) женщин. Медиана возраста в общей выборке пациентов составила 55,5 [52,54-58,52] года; в группе пациентов с сахарным диабетом 1 типа 43,15 [36,35-50,63] года, в группе пациентов с сахарным диабетом 2 типа - 59,91 [57,94-61,62] года. Группы пациентов с сахарным диабетом 1 и 2 типов были сопоставимы по стажу заболевания (табл. 1).

Основные результаты исследования

Средний уровень гликированного гемоглобина среди всех включенных в исследование пациентов составил 9,29 [8,75-9,81]\%; медиана индекса массы тела 32,41 [30,78-34,31] кг/м². Пациенты с сахарным диабетом 2 типа имели достоверно более высокий уровень гликированного гемоглобина $(p=0,017)$ и более высокий индекс массы тела ( $p<0,0001)$, чем пациенты с сахарным диабетом 1 типа. Так, средний уровень гликированного гемоглобина среди пациентов с сахарным диабетом 1 типа составил 8,40 [7,60-9,19]\%; средний индекс массы тела - 25,03 [22,95-27,46] кг/м². В группе пациентов с сахарным диабетом 2 типа уровень гликированного гемоглобина составил в среднем 9,61 [9,07-10,27]\%; индекс массы тела - 35,05 [33,25-36,98] кг/м². Средняя СКФ достоверно не различалась в группах с сахарным диабетом 1 и 2 типа и соответствовала ХБП стадии С2. Микроальбуминурия в среднем была выше в группе пациентов с сахарным диабетом 1 типа, чем у пациентов с сахарным диабетом 2 типа, но различия были статистически не значимы $(p=0,177)$.

Клиническая характеристика групп, включенных в исследование, представлена в таблице 1.

Таблица 1. Клиническая характеристика групп

\begin{tabular}{lcc}
\hline \multicolumn{1}{c}{ Показатель } & Сахарный диабет 1 типа & Сахарный диабет 2 типа \\
\hline Возраст, лет & $43,15[36,35-50,63]$ & $59,91[57,94-61,62]^{*}$ \\
Длительность заболевания, лет & $10,23[7,06-13,12]$ & $11,04[9,48-12,56]$ \\
Индекс массы тела, кг/м² & $25,03[22,95-27,46]$ & $35,05[33,25-36,98]^{*}$ \\
Гликированный гемоглобин, \% & $8,40[7,60-9,19]$ & $9,61[9,07-10,27]^{*}$ \\
СКФ, мл/мин & $80,16[66,71-93,71]$ & $75,81[70,39-81,04]$ \\
МАУ, Мг/л & $100,71[34,48-187,99]$ & $43,92[18,68-75,01]$ \\
АСТ, ЕД/л & $22,41[17,76-21,86]$ & $27,10[21,10-36,50]$ \\
АЛТ, ЕД/л & $20,24[17,50-22,77]$ & $25,04[20,60-30,87]$
\end{tabular}


Таблица 2. Сравнительная характеристика параметров феррокинетики и маркеров воспаления у пациентов с сахарным диабетом 1 и 2 типов

\begin{tabular}{lcc}
\hline \multicolumn{1}{c}{ Показатель } & Сахарный диабет 1 типа & Сахарный диабет 2 типа \\
\hline Гемоглобин, г/л & $133,25 \pm 4,45$ & $136,81 \pm 2,47$ \\
Эритроциты, $\times 10^{12} / л$ & $4,73 \pm 0,13$ & $4,84 \pm 0,08$ \\
Ретикулоциты, \% & $1,52 \pm 0,21$ & $2,20 \pm 0,40$ \\
Гематокрит, \% & $39,88 \pm 1,39$ & $41,73 \pm 0,70$ \\
Железо, мкмоль/л & $12,85 \pm 1,76$ & $16,23 \pm 1,32$ \\
Ферритин, нг/мл & $110,59 \pm 27,06$ & $149,68 \pm 18,06$ \\
Гепсидин, пг/мл & $8407,57 \pm 1124,72$ & $7457,96 \pm 672,49$ \\
СОЭ, мм/ч & $18,80 \pm 3,58$ & $19,83 \pm 1,81$ \\
Лейкоциты, $\times 10^{9} / л$ & $6,57 \pm 0,37$ & $7,67 \pm 0,27^{*}$ \\
СРБ, нг/мл & $4,64 \pm 1,53$ & $10,89 \pm 2,46^{*}$ \\
\hline
\end{tabular}

${ }^{*} p<0,05$

Параметры феррокинетики - гемоглобин, количество эритроцитов, ретикулоцитов, уровень гематокрита, ферритин, железо сыворотки и гепсидин - значимо не различались у пациентов с сахарным диабетом 1 и 2 типов (табл. 2). При этом в группе пациентов с сахарным диабетом 2 типа уровни СРБ $(p=0,034)$ и лейкоцитов крови ( $p=0,020)$ были достоверно выше, чем у пациентов с сахарным диабетом 1 типа (см. табл. 2).

Для установления конкретного вида нарушения феррокинетики (железодефицитной анемии, анемии хронических болезней или синдрома дисметаболической перегрузки железом) использовались указанные выше клинические рекомендации. Однако в 35,1\% случаев наблюдалась диссоциация параметров, используемых в качестве критериев диагностики (железо сыворотки, ферритин, гепсидин), и достоверно дифференцировать вид нарушения феррокинетики удалось только в остальных 64,9\% случаев. Диагноз анемии и ее вид (железодефицитная/анемия хронических заболеваний) устанавливались в этом случае на основании диагностических критериев - уровень гемоглобина, содержание железа сыворотки, уровень ферритина крови; уровень гепсидина для установления типа ане- мии в связи с диссоциацией параметров диагностики не учитывался.

Характеристика структуры анемического синдрома в исследуемых группах представлена в таблице 3 .

Таким образом, как в общей выборке пациентов с нарушениями углеводного обмена, так и у пациентов с сахарным диабетом 2 типа в структуре анемического синдрома преобладала анемия хронических заболеваний. Следует отметить, что только у одного пациента из всей выборки имелись признаки перегрузки железом - повышение уровня железа до 60 мкмоль/л и ферритина до 500 нг/мл при нормальных значениях гемоглобина и гематокрита; данный пациент страдал сахарным диабетом 2 типа, у него также отмечалось значительное повышение активности трансаминаз - аспартатаминотрансфераза (АСТ) 200 ЕД/л, аланинаминотрансфераза (АЛТ) 120 ЕД/л. В других случаях критериев перегрузки железом выявлено не было.

После разделения общей выборки пациентов на группы по виду анемического синдрома - отсутствие анемии, анемия хронических заболеваний и железодефицитная анемия - был проведен сравнительный анализ средних значений маркеров воспаления и уровня гепсидина в данных группах (табл. 4).

Таблица 3. Структура анемического синдрома у пациентов с сахарным диабетом 1 и 2 типа

\begin{tabular}{|c|c|c|c|}
\hline Наличие и вид анемии & $\begin{array}{c}\text { Общая выборка } \\
\text { пациентов } \\
(\mathbf{n}=76)\end{array}$ & $\begin{array}{c}\text { Сахарный диабет } \\
1 \text { типа } \\
(n=20)\end{array}$ & $\begin{array}{c}\text { Сахарный диабет } \\
2 \text { типа } \\
(n=56)\end{array}$ \\
\hline Железодефицитная анемия, \% (n) & $15,8(12)$ & $30,0(6)$ & $10,7(6)$ \\
\hline Анемия хронических заболеваний, \% (n) & $38,1(29)$ & $30,0(6)$ & $41,1(23)$ \\
\hline Отсутствие анемии, \% (n) & $46,1(35)$ & $40(8)$ & $48,2(27)$ \\
\hline
\end{tabular}

Таблица 4. Концентрация гепсидина и неспецифических маркеров воспаления в зависимости от наличия и вида анемии

\begin{tabular}{lccc}
\hline \multicolumn{1}{c}{ Показатель } & $\begin{array}{c}\text { Анемия хронических } \\
\text { заболеваний }\end{array}$ & $\begin{array}{c}\text { Железодефицитная } \\
\text { анемия }\end{array}$ & Нет анемии \\
\hline Гепсидин, пг/мл & $8324,59 \pm 959,78^{*}$ & $110606,30 \pm 1440,49^{*}$ & $6151,52 \pm 723,99$ \\
СОЭ, Мм/4 & $27,25 \pm 3,12^{*}$ & $18,58 \pm 3,47$ & $13,56 \pm 1,49$ \\
СРБ, нг/мл & $14,37 \pm 4,56$ & $5,28 \pm 1,46$ & $6,16 \pm 0,93$ \\
Лейкоциты, $\times 10^{9} / л$ & $8,00 \pm 0,39$ & $6,87 \pm 0,62$ & $7,04 \pm 0,30$ \\
\hline
\end{tabular}

*p<0,05 - относительно пациентов без анемии 
Было установлено, что у пациентов с анемией хронических заболеваний уровень гепсидина достоверно выше, чем у пациентов без анемического синдрома $(\mathrm{p}=0,033)$. Однако у пациентов с железодефицитной анемией уровень гепсидина был также достоверно выше, чем у пациентов без анемического синдрома $(p=0,009)$, и выше, чем в случае анемии хронических заболеваний, что несколько отличается от данных литературы [3, 4], в том числе расходится с данными, представленными в национальных клинических рекомендациях $[8,9]$. Достоверных различий в уровне СРБ между группами в зависимости от наличия и вида анемического синдрома получено не было, но наблюдались более высокие значения данного маркера воспаления у пациентов с анемией хронических заболеваний в сравнении как с пациентами без анемии $(p=0,058)$, так и с пациентами с железодефицитной анемией $(p=0,066)$. Кроме того, в группе анемии хронических заболеваний были достоверно выше значения СОЭ в сравнении с пациентами без анемического синдрома ( $<<0,0001)$. СОЭ в группах анемии хронических заболеваний и железодефицитной анемии также различались и была выше в первом случае, но достоверность различий не была достигнута $(p=0,074)$. В группе пациентов без анемии значения СОЭ были также ниже, чем у пациентов с железодефицитной анемией, но без статистической достоверности ( $\mathrm{p}=0,203)$. Таким образом, в случае наличия анемического синдрома имело место повышение значений маркеров воспаления, таких как СРБ, СОЭ и гепсидин, наиболее выраженное при анемии хронических заболеваний.

Был проведен парный корреляционный анализ как в общей выборке пациентов, так и в отдельных группах пациентов с сахарным диабетом 1 и 2 типов. В результате парного корреляционного анализа в общей выборке пациентов была выявлена положительная корреляционная связь уровня гепсидина с длительностью заболевания $(r=0,260 ; p=0,028)$, что характеризует повышение риска развития анемии хронических заболеваний с увеличением длительности сахарного диабета. Со скоростью оседания эритроцитов положительно коррелировали микроальбуминурия $(r=0,515 ; p<0,0001)$, концентрация креатинина $(r=0,467 ; p<0,0001)$ и отрицательно коррелировали СКФ ( $r=-0,436 ; p<0,0001)$ и содержание сывороточного железа ( $r=-0,276 ; p=0,017)$. Данные корреляционные связи отражают роль нарушения функции почек, прогрессирующего с течением времени при длительно текущем диабете, в развитии системного воспаления и анемии. Микроальбуминурия отрицательно коррелировала также с содержанием эритроцитов ( $r=-0,370$; $\mathrm{p}=0,002)$ и уровнем гемоглобина $(r=-0,295 ; p=0,013)$, что отражает роль нарушения функции почек в развитии анемического синдрома. Кроме того, отмечалась положительная корреляционная связь уровня ферритина с активностью трансаминаз - АСТ $(r=0,455 ; p<0,0001)$ и АЛТ $(r=0,451 ; p<0,0001)$ и концентрацией СРБ ( $r=0,310$; $\mathrm{p}=0,008)$, что отражает роль нарушения функции печени в развитии системного воспаления и дисметаболической перегрузки железом. Содержание железа сыворотки также положительно коррелировало с активностью как АСТ $(r=0,489 ; p<0,0001)$, так и АЛТ ( $r=0,498 ; p<0,0001)$.

Парный корреляционный анализ в группе пациентов с сахарным диабетом 2 типа также выявил наличие положительной корреляции значений СОЭ с микроальбуминурией $(r=0,412 ; p=0,002)$ и содержанием креатинина $(r=0,458 ; p=0,001)$, отрицательной - с СКФ ( $r=-0,368$; $\mathrm{p}=0,006)$, содержанием железа сыворотки ( $r=-0,283$; $p=0,038)$, количеством эритроцитов $(r=-0,639 ; p<0,0001)$, уровнем гемоглобина $(r=-0,453 ; p=0,001)$ и гематокрита $(r=-0,472 ; p=0,001)$. Данные корреляционные связи отражают роль нарушения функции почек, прогрессирующего при длительно текущем диабете, в развитии системного воспаления и анемии. В группе пациентов с сахарным диабетом 2 типа, как и в общей выборке пациентов, концентрация ферритина положительно коррелировала с активностью трансаминаз крови - АСТ $(r=0,505 ; p<0,0001)$ и АЛТ $(r=0,442 ; p=0,002)$. Уровень железа сыворотки также положительно коррелировал с активностью как АСТ ( $r=0,560 ; p<0,0001)$, так и АЛТ ( $r=0,559$; $\mathrm{p}<0,0001)$, что подтверждает роль нарушения функции печени в развитии системного воспалительного ответа и синдрома дисметаболической перегрузки железом.

В группе пациентов с сахарным диабетом 1 типа были выявлены аналогичные корреляционные связи. Микроальбуминурия положительно коррелировала с СОЭ $(r=0,697 ; p=0,001)$ и количеством лейкоцитов $(r=0,615$; $\mathrm{p}=0,005)$ и отрицательно - с количеством эритроцитов $(r=-0,510 ; p=0,026)$. СОЭ положительно коррелировала с концентрацией креатинина $(r=0,501 ; p=0,024)$, отрицательно - СКФ ( $r=-0,553 ; p=0,012)$. Кроме того, были выявлены корреляционные связи СОЭ с параметрами феррокинетики: положительная связь с количеством ретикулоцитов крови ( $r=0,632 ; p=0,05)$, отрицательная корреляционная связь с количеством эритроцитов $(r=-0,768$; $p<0,0001)$, гемоглобина $(r=-0,527 ; p=0,017)$ и гематокритом ( $r=-0,556 ; p=0,021)$, что отражает роль системного воспаления в развитии анемического синдрома у пациентов с нарушениями углеводного обмена. Корреляционных связей параметров феррокинетики (ферритин, железо сыворотки) с активностью трансаминаз в группе пациентов с сахарным диабетом 1 типа выявлено не было.

Был проведен ROC-анализ, по результатам которого наибольшую информативность в диагностике анемии хронических заболеваний в общей выборке пациентов показали: ферритин - чувствительность 78\%, специфичность 52\% при диагностическом пороге 75,5 нг/мл (площадь под кривой 0,695; $\mathrm{p}=0,006)$; СОЭ - чувствительность 67\%, специфичность 64\% при диагностическом пороге 15,5 мм/ч (площадь под кривой 0,750; $\mathrm{p}=0,040$ ) и СРБ - чувствительность 67\%, специфичность 64\% при диагностическом пороге 5,2 нг/мл (площадь под кривой 0,646; р<0,0001) (рис. 1).

В группе пациентов с сахарным диабетом 2 типа по результатам ROC-анализа наибольшей информативностью в диагностике анемии хронических заболеваний обладали те же показатели: ферритин - чувствительность 71\%, специфичность 62\% при диагностическом пороге 99,7 нг/мл (площадь под кривой 0,700; $p=0,0017$ ) (рис. 3); СОЭ - чувствительность 67\%, специфичность 59\% при диагностическом пороге 17,5 мм/ч (площадь под кривой 0,$731 ; p=0,006)$ (рис. 2) и СРБ - чувствительность 71\%, специфичность 55\% при диагностическом пороге 5,6 нг/мл (площадь под кривой 0,644; $\mathrm{p}=0,085$ ) (рис. 2), а также гепсидин - чувствительность 
71\%, специфичность 52\% при диагностическом пороге 6545,5 пг/мл (площадь под кривой 0,631; $p=0,082$ ) (рис. 3).

Согласно результатам ROC-анализа, проведенного в группе пациентов с сахарным диабетом 1 типа, высокую информативность в диагностике анемии хронических заболеваний имели СОЭ - чувствительность 83\%, специфичность 54\% при диагностическом пороге 12,5 мм/ч (площадь под кривой 0,763; $p=0,076$ ) и ферритин - чувствительность 83\%, специфичность 54\% при

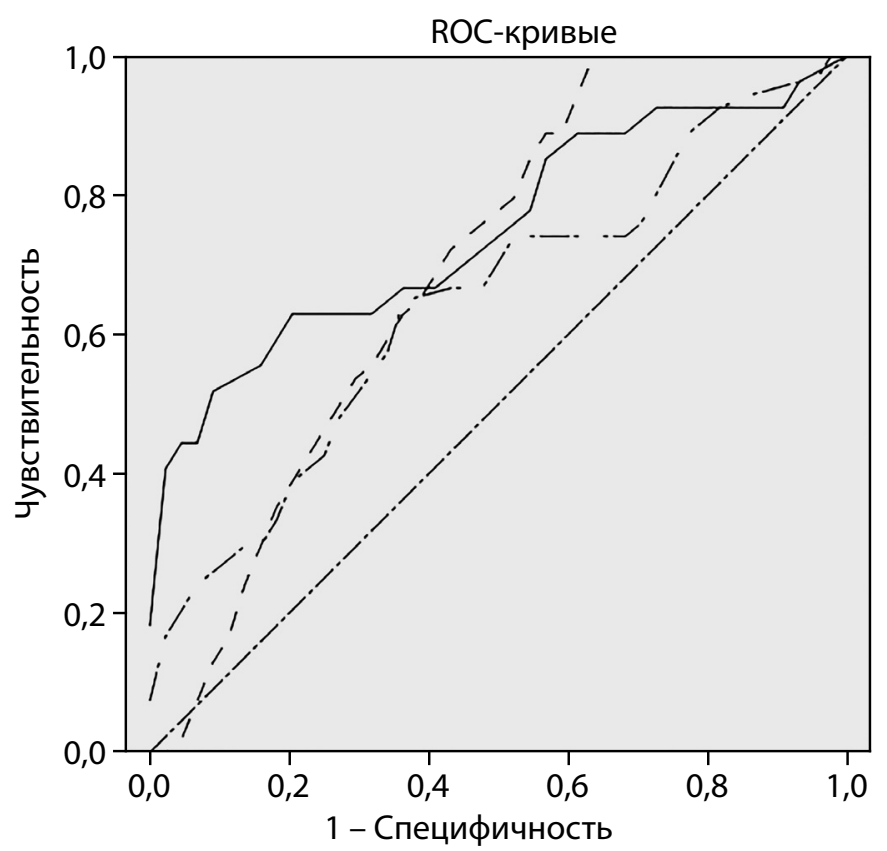

Диагональные сегменты, сгенерированные связями

\section{— СOЭ -—- CRP - - Ферритин --ООпорная линия}

Рисунок 1. Информативность ферритина, С-реактивного белка и скорости оседания эритроцитов в диагностике анемии хронических заболеваний при сахарном диабете

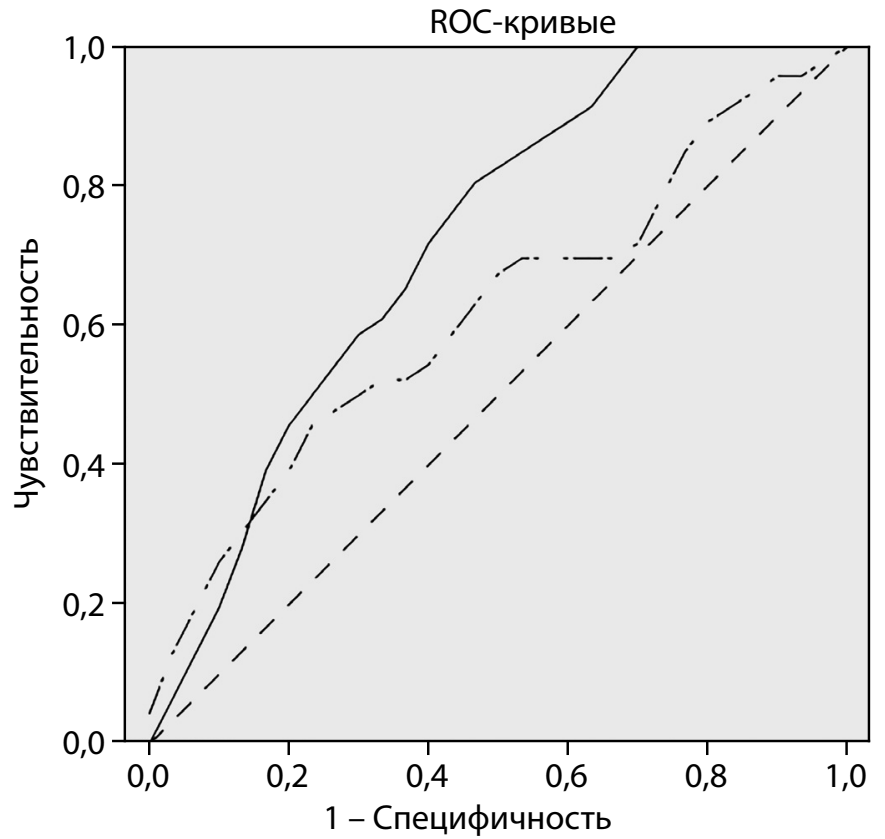

Диагональные сегменты, сгенерированные связями - Ферритин -- Гепсидин - - Опорная линия

Рисунок 3. Информативность ферритина и гепсидина в диагностике анемии хронических заболеваний при сахарном диабете 2 типа диагностическом пороге 44,5 нг/мл (площадь под кривой 0,$667 ; p=0,187$ ) (рис. 4).

Таким образом, исследованные маркеры воспаления СОЭ и СРБ, а также гепсидин в совокупности с классическим диагностическим параметром - ферритином продемонстрировали высокую диагностическую ценность в отношении анемии хронических заболеваний и могут быть внесены в модифицированный алгоритм дифференциальной диагностики анемического синдрома.

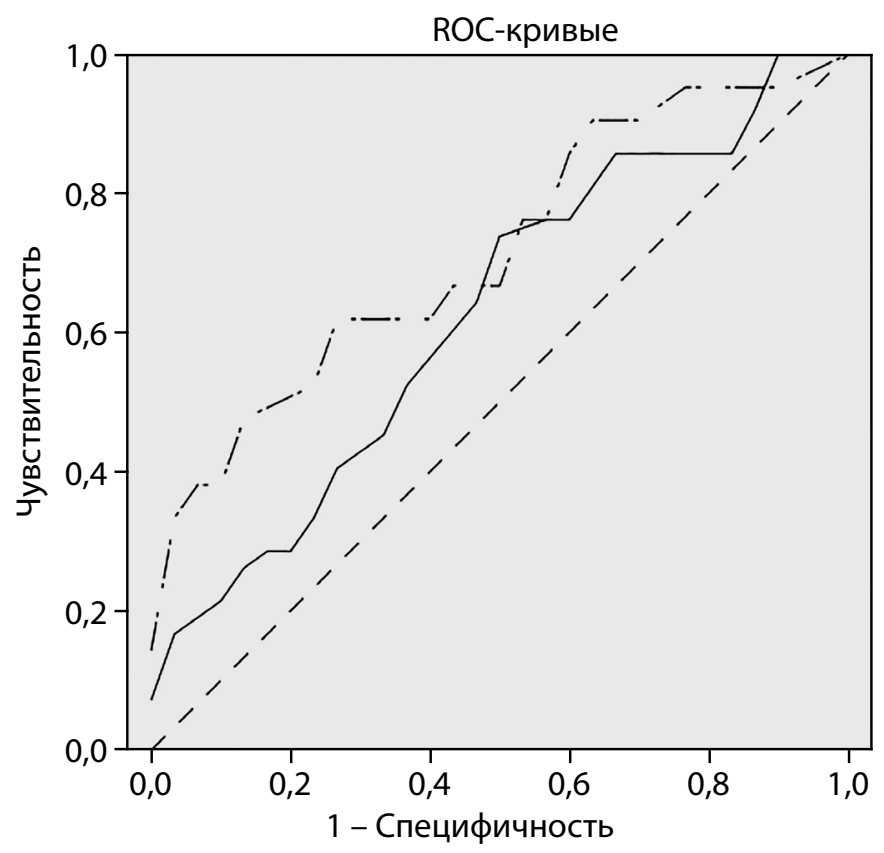

Диагональные сегменты, сгенерированные связями

$$
\text { - CRP - - СOЭ - - Опорная линия }
$$

Рисунок 2. Информативность С-реактивного белка и скорости оседания эритроцитов в диагностике анемии хронических заболеваний при сахарном диабете 2 типа

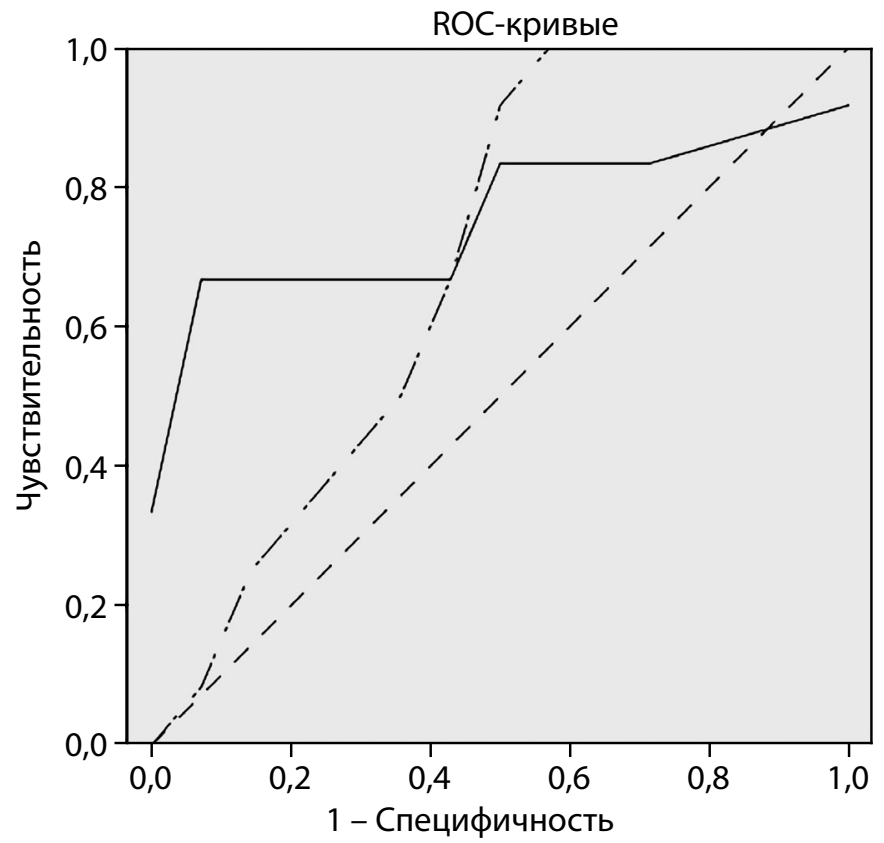

Диагональные сегменты, сгенерированные связями

$$
\text { — СОэ --. Ферритин - - Опорная линия }
$$

Рисунок 4. Информативность ферритина и скорости оседания эритроцитов в диагностике анемии хронических заболеваний у пациентов с сахарным диабетом 1 типа 


\section{ОБСУЖДЕНИЕ}

Резюме и обсуждение основного результата

\section{исследования}

В результате проведенного исследования было установлено, что среди обследованных пациентов сахарный диабет как 1, так и 2 типа более чем в 50\% случаев сопровождается развитием анемического синдрома, при этом в структуре анемии при сахарном диабете 2 типа преобладает анемия хронических заболеваний. Данный вид анемии у пациентов с сахарным диабетом был ассоциирован с повышением маркеров воспаления - СОЭ, СРБ и количества лейкоцитов, а также с повышением содержания гепсидина крови. Согласно данным литературы, распространенность анемического синдрома у пациентов с сахарным диабетом несколько ниже $[10,11]$. Так, по результатам исследования, проведенного в 2010 г. в республике Татарстан, частота развития анемического синдрома у пациентов с сахарным диабетом 1 типа составила 34\%, при этом вид анемии зависел от наличия и выраженности диабетической нефропатии - у пациентов с сохраненной функцией почек и без клинических проявлений диабетической нефропатии преобладала железодефицитная анемия, у лиц со сниженной функцией почек - анемия хронических заболеваний [12]. Кроме того, в данном исследовании также имела место диссоциация параметров дифференциальной диагностики, а практически каждый третий случай анемии был расценен как сочетание анемии хронических заболеваний и истинного железодефицита [12]. По данным опубликованного в 2019 г. исследования, проведенного на базе БУЗ ВО «ВГКБСМП №10» г. Воронежа, частота встречаемости анемии в когорте пациентов с сахарным диабетом 2 типа составила 28,2\%, структура анемического синдрома при этом не изучалась, но было отмечено, что у пациентов с хронической болезнью почек 1-3 стадии частота анемии была несколько выше и составляла 30,7\% [13]. Масштабное исследование, проведенное в 2015 г. на базе «Мобильного Диабет-центра» в рамках Федеральной целевой программы «Сахарный диабет», также показало, что распространенность анемии была выше в группах пациентов с хронической болезнью почек и составила $38,8 \%$ и 22,6\% соответственно у пациентов с 1 и 2 типами сахарного диабета [14]. Изучение структуры анемического синдрома при этом не было целью исследования, а исследовалась зависимость частоты и тяжести анемии от наличия и стадии хронической болезни почек, в том числе микроальбуминурии. Так, было показано, что у больных с диабетическим поражением почек частота выявления анемии превышает таковую у больных с недиабетической почечной патологией без различия в степени ее тяжести [14]. Аналогичное исследование частоты анемии у пациентов с сахарным диабетом было проведено в Кувейте и опубликовано в 2017 г. [15]. Согласно результатам исследования, частота анемии у пациентов с сахарным диабетом составила 28,5\% и была выше среди женщин (35,8\%), чем среди мужчин $(21,3 \%)$; было также показано, что частота развития анемии коррелировала с уровнями микроальбуминурии и креатинина и не зависела от гликемического контроля [15]. В целом стоит отметить, что в настоящее время как в России, так и за рубежом опубликовано довольно небольшое количество работ, посвященных изучению структуры анемического синдрома у пациентов с нарушениями углеводного обмена и информативности диагностических критериев анемии при сахарном диабете.

В нашем исследовании было выявлено наличие положительной корреляционной связи между уровнем гепсидина и стажем сахарного диабета, что характеризует повышение риска развития анемии хронических заболеваний с увеличением длительности диабета. В обзоре литературы, опубликованном в 2018 г., приведен подробный анализ данных зарубежной литературы относительно механизмов регуляции продукции гепсидина и нарушения обмена данного пептида при различных нозологиях, в том числе сахарном диабете [16]. Как уже отмечалось, сахарный диабет и ожирение сопровождаются хроническим системным воспалением и повышением уровней IL-1 $\beta$, IL-6 и СРБ, участвующих в активации сигнального пути с повышением экспрессии гена НАМР и синтеза гепсидина, приводя таким образом к развитию анемии хронических заболеваний $[5,17]$. Поскольку хроническая болезнь почек сопровождается наличием воспаления, продукция IL-6 и IL-1 $\beta$ поддерживает активацию сигнального пути JAK/STAT, повышая транскрипцию гена HAMP и, соответственно, синтез гепсидина. С развитием хронической болезни почек также нарушается клиренс самого гепсидина и повышается его содержание в плазме крови, ограничивая таким образом биодоступность железа и усугубляя течение анемии хронических заболеваний [7]. Эти данные соотносятся с полученными в нашем исследовании результатами. Так, было выявлено наличие положительных корреляционных связей маркеров воспаления с креатинином и микроальбуминурией и отрицательных со скоростью клубочковой фильтрации и такими параметрами феррокинетики, как железо сыворотки и уровень гемоглобина, что отражает роль прогрессирующего при длительном течении диабета нарушения функции почек в развитии системного воспаления и анемии. Выявленная в нашем исследовании сильная положительная корреляционная связь между уровнем активности печеночных трансаминаз, ферритином и железом сыворотки крови у пациентов с сахарным диабетом 2 типа, а также с уровнем СРБ подтверждает роль нарушения функции печени в развитии системного воспаления и синдрома дисметаболической перегрузки железом. Согласно литературным данным, перегрузка железом с его избыточным отложением в печени часто сопровождается инсулинорезистентностью и предрасполагает к развитию сахарного диабета 2 типа [18]. Несомненно, целесообразными представляются изучение некоторых других маркеров воспаления - IL-1 $\beta$ и IL-6, играющих роль в патогенезе анемии хронических заболеваний, и уточнение их информативности в дифференциальной диагностике анемического синдрома у лиц с нарушениями углеводного обмена. Также планируется расширение выборки пациентов с добавлением групп сравнения (пациенты с ожирением без нарушений углеводного обмена) и контроля (здоровые добровольцы без анемии).

Ограничения исследования

В данном исследовании выделить группу пациентов с дисметаболической перегрузкой железом на фоне нарушений углеводного обмена не удалось, вероятно, 
ввиду относительно небольшого объема выборки. Определение классических параметров дифференциальной диагностики анемии хронических заболеваний - общей и латентной железосвязывающей способности сыворотки не было целью исследования. Поскольку в исследование не включались пациенты с СКФ ниже 15 мл/мин, оценка уровня эритропоэтина не представлялась целесообразной. Трансферрин, являющийся одним из общепринятых параметров диагностики анемии хронических заболеваний, в ходе данной работы не определялся ввиду отсутствия на момент проведения исследования реактивов, однако оценка информативности трансферрина и растворимых рецепторов к трансферрину планируется в продолжении исследования.

\section{ЗАКЛЮЧЕНИЕ}

Таким образом, результаты нашего исследования позволяют предположить, что нарушения функции печени, почек и развитие системного субклинического воспаления при сахарном диабете являются значимыми факторами риска нарушений феррокинетики и могут затруднять дифференциальную диагностику между железодефицитной анемией и анемией хронических заболеваний. Проведенный ROC-анализ продемонстрировал высокую информативность определения таких параметров, как гепсидин, СОЭ и СРБ, в диагностике анемии хронических заболеваний. Данные маркеры, наряду с классическим диагностическим параметром - ферритином, могут быть включены в алгоритм дифференциальной диагностики анемического синдрома у пациентов с нарушениями углеводного обмена.

\section{ДОПОЛНИТЕЛЬНАЯ ИНФОРМАЦИЯ}

Источник финансирования. Исследование выполнено при финансовой поддержке гранта РФФИ Аспиранты №19-315-90061 «Взаимосвязь нарушений феррокинетики и метаболических изменений при сахарном диабете».

Конфликт интересов. Авторы декларируют отсутствие явных и потенциальных конфликтов интересов, связанных с публикацией настоящей статьи.

Участие авторов. Мусина Н.Н. - разработка концепции и дизайна исследования; сбор материала; анализ и интерпретация данных; подготовка текста статьи; Саприна Т.В. - разработка концепции и дизайна; обоснование рукописи и проверка критически важного интеллектуального содержания; окончательное утверждение для публикации рукописи; Прохоренко Т.С. - сбор материала; обоснование рукописи и проверка критически важного интеллектуального содержания; Зима А.П. - обоснование рукописи и проверка критически важного интеллектуального содержания. Все авторы внесли значимый вклад в проведение исследования и подготовку статьи, прочли и одобрили финальную версию статьи перед публикацией.

\section{СПИСОК ЛИТЕРАТУРЫ | REFERENCES}

1. Fernandez-Real JM, Lopez-Bermejo A, Ricart W. Cross-talk between iron metabolism and diabetes. Diabetes. 2002;51(8):2348-2354. doi: 10.2337/diabetes.51.8.2348.

2. Sam AH, Busbridge $M$, Amin $A$, et al. Hepcidin levels in diabetes mellitus and polycystic ovary syndrome. Diabet Med. 2013;30(12):1495-1499. doi: 10.1111/dme.12262.

3. Jiang F, Sun ZZ, Tang YT, et al. Hepcidin expression and iron parameters change in type 2 diabetic patients. Diabetes Res Clin Pract. 2011;93(1):43-48. doi: 10.1016/j.diabres.2011.03.028.

4. Aregbesola A, Voutilainen S, Virtanen JK, et al. Serum hepcidin concentrations and type 2 diabetes. World J Diabetes. 2015;6(7):978-982. doi: 10.4239/wjd.v6.i7.978.

5. Ganz T. Hepcidin and iron regulation, 10 years later. Blood. 2011;117(17):4425-4433. doi: 10.1182/blood-2011-01-258467.

6. Jiang F, Sun ZZ, Tang YT, et al. Hepcidin expression and iron parameters change in type 2 diabetic patients. Diab Res Clin Pract. 2011;93(1):43-48. doi: 10.1016/j.diabres.2011.03.028.

7. Ganz T, Nemeth E. Hepcidin and iron homeostasis. Biochim Biophys Acta. 2012;1823(9):1434-1443. doi: 10.1016/j.bbamcr.2012.01.014.

8. Румянцев А.Г., Масчан А.А., Чернов В.М., Тарасова И.С. Федеральные клинические рекомендации по диагностике и лечению железодефицитной анемии. - M., 2015. - 43 с. [Rumyantsev AG, Maschan AA, Chernov VM, Tarasova IS. Federal 'nye klinicheskie rekomendatsii po diagnostike i lecheniyu zhelezodefitsitnoy anemii. Moscow; 2015. 43 p. (In Russ).]

9. Румянцев А.Г., Масчан А.А. Федеральные клинические рекомендации по диагностике и лечению анемии хронических болезней. - M., 2014. - 9 c. [Rumyantsev AG, Maschan AA. Federal nye klinicheskie rekomendatsii po diagnostike i lecheniyu anemii khronicheskikh bolezney. Moscow; 2014. 9 p. (In Russ).]

10. Thomas M, Tsalamandris C, Maclsaac R, Jerums G. Anemia in diabetes: an emerging complication of microvascular disease. Curr Diabetes Rev. 2005;1(1):107-126. doi: 10.2174/1573399052952587.

11. Angelousi A, Larger E. Anemia, a common but often unrecognized risk in diabetic patients: a review. Diabetes Metab. 2015;41(1):18-27. doi: 10.1016/j.diabet.2014.06.001
12. уфелкина Т.Ю., Валеева Ф.В. Анемия у больных сахарным диабетом 1 типа // Сахарный диабет. - 2010. - №4. C. 49-53. [Kufelkina TYu, Valeeva FV. Anemia in patients with type 1 diabetes mellitus. Diabetes mellitus. 2010;(4):49-53. (In Russ).]

13. Семакова А.Д., Брыкова Я.И., Силина М.Н., Волынкина А.П. Оценка распространенности анемии у больных с сахарным диабетом // Центральный научный вестник. - 2019. - Т.4. №7. - C. 7-8. [Semakova AD, Brykova Yal, Silina MN, Volynkina AP. Estimation of the anemia prevalence in patients with diabetes mellitus. Central Scientific Herald. 2019;4(7):7-8. (In Russ).]

14. Мартынов С.А., Шестакова М.В., Шилов Е.М., и др. Распространенность анемии у больных сахарным диабетом 1 и 2 типа с поражением почек // Сахарный диабет. 2017. - T.20. - №5. - C. 318-328. [Martynov SA, Shestakova MV, Shilov EM, et al. Prevalence of anemia in patients with type 1 and type 2 diabetes mellitus with chronic renal disease. Diabetes mellitus. 2017;20(5):318-328. (In Russ).] doi: 10.14341/DM9369.

15. Alsayegh F, Waheedi M, Bayoud T, et al. Anemia in diabetes: experience of a single treatment center in Kuwait. Prim Care Diabetes. 2017;11(4):383-388. doi: 10.1016/j.pcd.2017.04.002.

16. Саприна Т.В., Зима А.П., Мусина Н.Н., и др. Патогенетические аспекты нарушения метаболизма гепсидина и феррокинетики при патологии углеводного обмена // Сахарный диабет. 2018. - T.21. — №6. - C. 506-512. [Saprina TV, Zima AP, Musina NN, et al. Pathogenetic aspects of hepcidin metabolism and ferrocinetics dysregulation in carbohydrate metabolism disorders. Diabetes mellitus. 2018;21(6):506-512. (In Russ).] doi: 10.14341/DM9378.

17. Nemeth E, Rivera S, Gabayan V, et al. IL-6 mediates hypoferremia of inflammation by inducing the synthesis of the iron regulatory hormone hepcidin. J Clin Invest. 2004;113(9):1271-1276. doi: $10.1172 / \mathrm{JCl} 20945$

18. Simcox JA, Mcclain DA. Iron and diabetes risk. Cell Metab. 2013;17(3):329-341. doi: 10.1016/j.cmet.2013.02.007.

Рукопись получена: 16.01.2020. Одобрена к публикации: 24.04.2020. Опубликована online: 28.07.2020. 


\section{ИНФОРМАЦИЯ ОБ АВТОРАХ [AUTHORS INFO]}

* Мусина Надежда Нурлановна, аспирант [Nadezhda N. Musina, MD, PhD student]; адрес: 634050, Томск, Московский тракт, д. 2 [address: 2, Moscowski Trakt, Tomsk, 634050 Russian Federation]; ORCID: http://orcid.org/0000-0001-7148-6739; eLibrary SPIN: 3468-8160; e-mail: nadiezhda-musina@mail.ru

Саприна Татьяна Владимировна, д.м.н., профессор [Tatiana V. Saprina, MD, PhD, Professor]; ORCID: http://orcid.org/0000-0001-9011-8720; eLibrary SPIN: 2841-2371; e-mail: tanja.v.saprina@mail.ru Зима Анастасия Павловна, д.М.Н., профессор [Anastasia P. Zima, MD, PhD, Professor]; ORCID: http://orcid.org/0000-0002-9034-7264; eLibrary SPIN: 5710-4547; e-mail: zima2302@gmail.com Прохоренко Татьяна Сергеевна, к.м.н., н.с. [Tatiana S. Prokhorenko, MD, PhD, research associate]; ORCID: http://orcid.org/0000-0002-8016-4755; eLibrary SPIN: 1495-4127; e-mail: mmikld.ssmu@gmail.com

\section{КАК ЦИТИРОВАТЬ:}

Мусина Н.Н., Саприна Т.В., Прохоренко Т.С., Зима А.П. Поиск дополнительных маркеров нарушения феррокинетики при сахарном диабете. — 2020. - Т. 66. — №2. — C. 61-70. doi: https://doi.org/10.14341/probl12277

\section{TO CITE THIS ARTICLE:}

Musina NN, Saprina TV, Zima AP, Prokhorenko TS Searching for additional markers of impaired iron metabolism in diabetes mellitus. Problems of Endocrinology. 2020;66(2):61-70. doi: https://doi.org/10.14341/probl12277 